\title{
Design, synthesis, molecular docking studies and anti-microbial activity of novel 1,2,3,4-tetrahydrocarbazole derivatives
}

\author{
*Sakinala Padmavathi and Madhukar Rajaram Tajne \\ Department of Pharmaceutical Sciences, RTM Nagpur University, Nagpur, Maharashtra, India- 440033
}

\begin{abstract}
The heterocyclic compounds naming tetrahydrocarbazoles having the significant biological properties. The newly substituted tetrahydrocarbazole derivatives were prepared from substituted phenylhydrazine and cyclohexanone in glacial acetic acid under reflux. These intermediates on reaction with substituted aromatic acid chlorides in alkaline media finally converted to N-Substituted tetrahydrocarbazoles. Fifteen compounds are synthesized and characterized by their melting point (MP), IR, NMR, MS and elemental analysis. All the compounds were subjected to molecular docking studies for Gln-6-p enzyme (1XFF) inhibition. The results of in silico molecular docking showed that all the derivates have significant binding energies, good affinity with active pocket and it may be reflected as a good inhibitor of GlcN-6-P synthase. The anti-microbial activity was assessed by agar cup plate method and the result showed 8 compounds having the better anti-microbial response against the bacterial and fungal strains. In conclusion, the study helps to give the greater scope of developing these tetrahydrocarbazoles derivatives which help to promote the effective anti-bacterial agents.
\end{abstract}

Key Words: Tetrahydrocarbazoles, fisher indole synthesis, molecular docking, anti-microbial activity.

\section{INTRODUCTION}

In general, the microbes of mesophiles causes the most of the pathogenic diseases in the animals include human beings. In the present situation most of the pathogens getting more resistant to anti-microbials so, it is considered as a major issue all around the world. Hence, there is a significant need to develop new anti-microbial agents to fight against life threatening invasive infections. As per the new concepts of drug design, docking studies help to understand in the better way about the interactions of drugs with receptor. (Hughes et al., 2011, Kapetanovic 2008, Meng et al., 2013). The enzyme- GlcN-6-P synthase or glucosamine-6phosphate synthase, is a new target for the drugs of antimicrobials (Vijesh et al., 2013). It is a protein synthesis inhibitor in bacteria. It binds to a small 16s rRNA of the 30s subunit of the ribosome in bacteria which interferes the binding of formyl-methionyl-tRNA to the 30s subunit.

The tetrahydrocarbazoles (figure 1) represents leading group of the ring system and have attracted a great deal of activites. It mainly acts up on the peptide cyclin dependent kinases inhibitors (CDKs) (Zhu et al., 2004), check point kinase inhibitors (Chk1) (Conchon et al., 2008), and peroxisome proliferation activated receptors (PPAR $\alpha, \beta$, and $\gamma$ ) (Kumar et al., 2005). In addition, tetrahydrocarbazoles possess more active to treat the neoplastic and cardiovascular disorders (Kaushik et al., 2012). Literature survey revealed that tetrahydrocarbazoles having the nitrogen atom having the rigid aromatic moiety helps to the electron transfer in the $\pi$-conjugated system (Chakraborty et al., 2014, Zhang et al., 2010) possess the wide range of pharmacological activities include anti-microbial activity, antipyretics antiinflammatory, antiproliferative, serum lipid lowering agent (Subramanyam et al., 2014, Ya-ching et al., 2005, Crosby et al., 1947).

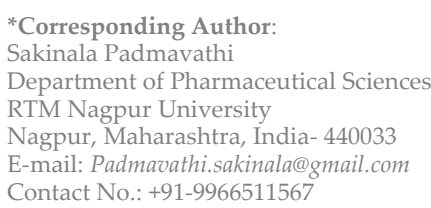

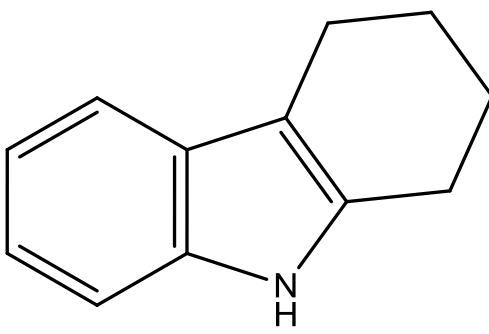

Figure 1: Structure of Tetrahydrocarbazole.

In the present study of tetrahydrocarbazoles, we are aimed to synthesize the tetrahydrocarbazole derivatives in an efficient manner. The objective of the study was to synthesize, characterize the $\mathrm{N}$-substituted tetrahydrocarbazole compounds along with molecular docking studies of the synthesized compounds in associated with the evaluation of anti-bacterial activity by agar cup method.

\section{MATERIALS AND METHODS}

All the chemicals for the synthetic work were used of analytical grade from SD Fine Chem Limited, Merck, Loba Chemie Private limited and Sigma Aldrich. The residue has been dried in vacuum desiccator and recrystallised from ethanol. The melting points of the compounds were determined in open capillaries using thiel's tube. Precoated silicagel- $\mathrm{G}$ plates are activated at $110^{\circ} \mathrm{C}$ for 30 minutes were used for thin layer chromatography and the spots were developed in iodine chamber. The mobile phase for the analysis of TLC was n-hexane: EtoAc $(7: 3 \mathrm{v} / \mathrm{v})$. The IR spectra of the compounds were recorded using pressed pellet technique using $\mathrm{KBr}$ on FTIR-8400 Spectrophotometer Shimadzu. ${ }^{1} \mathrm{H}-\mathrm{NMR}$ and ${ }^{13} \mathrm{C}$ Spectra $\left(\mathrm{CDCl}_{3}, \mathrm{DMSO}\right)$ were recorded on bruker advance-11, 400 Spectrophotometer on $400 \mathrm{MHz}$ at Loyola index laboratories using TMS as internal standard. The chemical shifts are reported in parts 
per million. Mass spectras were recorded using Waters Instrument having Q-TOF-MS. Elemental analysis was carried out SAIF Punjab University, Chandigarh.

\section{General Procedure}

\section{Synthesis of tetrahydrocarbazole}

The synthesis of the tetrehydrocarbazole was carried out based up on the Fischer indole synthesis (Colin et al., 2006) and the scheme was showed in figure 2 .

Scheme 1: Synthesis of substituted tetrahydrocarbazole Dissolve $8.8 \mathrm{gm}(0.08 \mathrm{~mol})$ of cyclohexanone in $50 \mathrm{ml}$ of glacial acetic acid add $8.8 \mathrm{gm}(0.08 \mathrm{~mol})$ of substituted phenyl hydrazine and boil the solution under reflux for $15 \mathrm{~min}$, cooled the solution, where the product was crystallised out. Filtered it with vacuum pump, then drained well and recrystallised using Ethanol.

\section{Scheme 2: Synthesis of N-substituted Tetrahydro- carbazoles}

Suspended $1 \mathrm{gm}(0.07 \mathrm{~mol})$ of substituted tetrahydrocarbazole in $20 \mathrm{~mL}$ of $10 \% \mathrm{NaOH}$ solution into a well corked conical flask and add $2 \mathrm{~mL}$ of substituted acid chloride with constant shaking for 10 minutes until the odour has disappeared and then it was cooled. Filtered and washed the synthesized N-Substituted derivative using water and recrystallized with ethanol.

\section{Computational Methods}

Molecular docking studies were carried out to explore binding modes of our derivatives with the target enzyme L-Glutamine: D -fructose-6-phosphate amido transferase. The molecular docking simulation studies were carried out by biopredicta tool of V Life MDS software version 4.2. The receptor employed here was Gln-6-p (PDB code 1XFF) obtained from RCSB protein data bank. The initial crystal structure consists of bound ligands it was removed and missing loops were added with help of homology modeling from the same software.

\section{Anti-bacterial activity}

Determination by Agar cup method

The antibacterial activity of 1,2,3,4-tetrahydrocarbazolederivatives was studied by agar cup method. The nutrient broth culture media was chosen as basal medium for testing the microbe. The nutrient broth medium (Hi media M0001) was plated into Petri dishes, allowed to solidification and then the microbe was inoculated into broth medium and allowed for incubation for a period of 24 hours at $25^{\circ} \mathrm{C}$. Bacterial culture was spread evenly over the entire surface to avoid the aggregation and left undisturbed for few minutes to permeate the culture. The wells/ holes $(4 \mathrm{~mm})$ were drawn using a sterile borer into the solidified nutrient medium. The compounds of substituted tetrhydrocarbazoles were added to each well $(100 \mu \mathrm{L})$ at peripheral of the petridish and the reference compounds (ciprofloxacin for bacterial, flucanazole for fungal) was added at the centre and then the plates are incubated for 24

\section{Scheme 1:}<smiles>[R]c1ccc2[nH]c3c(c2c1)CCCC3</smiles>

Phenylhydrazine

\section{Scheme 2:}
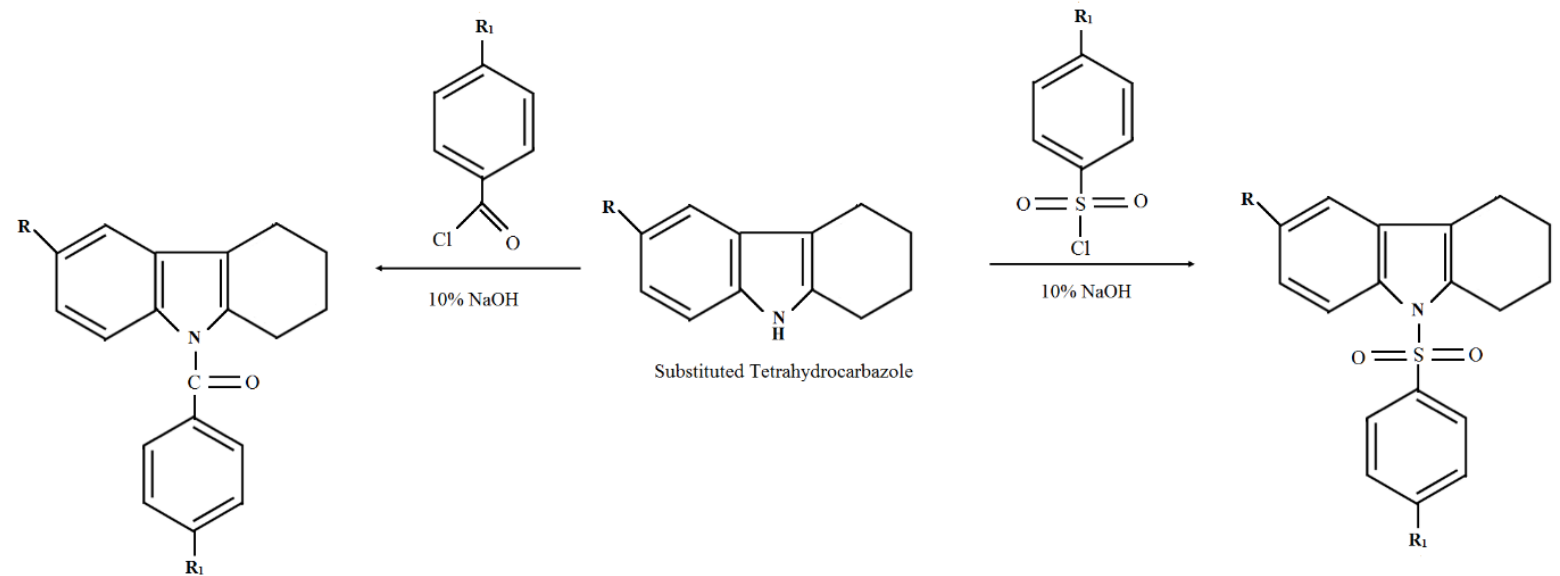

\begin{tabular}{|c|c|c|c|c|c|c|c|c|c|c|c|c|c|c|c|}
\hline & $1 \mathrm{a}$ & $1 \mathrm{~b}$ & $1 \mathrm{c}$ & $1 \mathrm{~d}$ & $2 \mathrm{a}$ & $2 \mathrm{~b}$ & $2 \mathrm{c}$ & $2 \mathrm{~d}$ & $3 \mathrm{a}$ & $3 \mathrm{~b}$ & $3 \mathrm{c}$ & $3 \mathrm{~d}$ & $3 \mathrm{e}$ & $3 \mathrm{f}$ & $3 \mathrm{~g}$ \\
\hline $\mathrm{R}$ & $\mathrm{H}$ & $\mathrm{Cl}$ & $\mathrm{F}$ & $\mathrm{CH}$ & $\mathrm{H}$ & $\mathrm{Cl}$ & $\mathrm{F}$ & $\mathrm{CH}_{3}$ & $\mathrm{H}$ & $\mathrm{Cl}$ & $\mathrm{F}$ & $\mathrm{CH}$ & $\mathrm{Cl}$ & $\mathrm{F}$ & $\mathrm{NO}$ \\
\hline $\mathrm{R}_{1}$ & $\mathrm{H}$ & $\mathrm{H}$ & $\mathrm{H}$ & $\mathrm{H}$ & $\mathrm{CH}_{3}$ & $\mathrm{CH}_{3}$ & $\mathrm{CH}_{3}$ & $\mathrm{CH}_{3}$ & $\mathrm{Cl}$ & $\mathrm{Cl}$ & $\mathrm{Cl}$ & $\mathrm{Cl}_{2}$ & $\mathrm{NO}_{2}$ & $\mathrm{NO}_{2}$ & $\mathrm{NO}_{2}$ \\
\hline
\end{tabular}

Figure 2: Scheme of 1,2,3,4 substituted Tetrahydrocarbazoles. 
hrs at $25^{\circ} \mathrm{C}$. The plates were collected and analyze the zone of inhibition with respect to millimeters $(\mathrm{mm})$ (Biswanath et al., 2014, Rajesh et al., 2015).

\section{RESULTS AND DISCUSSION}

By adopting above methodology the following mono, disubstituted tetrahydracarbazole were synthesized and it was showed in the scheme 1 and 2.

The synthesized tetrahydracarbazoles were analyzed using spectroscopic techniques. In the IR spectra the aromatic skeleton of the tetrahydrocarbazole appears at region of $1432-1630 \mathrm{~cm}^{-1}$ and characteristic $-\mathrm{NH}$ streching 3413$3410 \mathrm{~cm}^{-1}, \mathrm{C}=\mathrm{O}$ adsorption at $1730-1750 \mathrm{~cm}^{-1}$ and $\mathrm{S}=\mathrm{O}$ adsorption peak at $1350-1405 \mathrm{~cm}^{-1}$. The proton magnetic resonance spectra all the signals are at the respective positions. All the synthesized compounds give corresponding sharp M+1 peak in the Mass spectroscopy. The details of the spectral data are given below:

\section{Spectral data:}

1a.2,3,4,9-Tetrahydro-1H-carbazole

Yield $91 \%$; mp 117 - $119{ }^{\circ} \mathrm{C}$ (ethanol); Rf0.39 (n - Hexane: EtoAC ,7:3); IR (KBr) cm-1:3314.12(N-H stretching),2954.8 (C-H Stretching aliphatic), 1623 (C=C Stretching),1513.7 (C$\mathrm{N}$ stretching) ; ${ }^{1} \mathrm{H}$ NMR $\left(\mathrm{CDCl}_{3}, 400 \mathrm{MHz}\right): 2.08(\mathrm{~m}, 2 \mathrm{H}$, $\left.\mathrm{ABq}, \mathrm{CH}_{2}\right), 2.20\left(\mathrm{~m}, 2 \mathrm{H}, \mathrm{ABq}, \mathrm{CH}_{2}\right), 2.28\left(\mathrm{~m}, 4 \mathrm{H}, \mathrm{CH}_{2}\right), 3.90$ (s, $1 \mathrm{H}, \mathrm{NH}), 6.73$ (m, 4H, Ar); ${ }^{13} \mathrm{CNMR}: 115.76,118.50,129$ (Ar), 168.16, 169.10, 170.12 (Tetrahydrocarbazole); Em (Es, Positive mode) $\mathrm{m} / \mathrm{z}$ 171.165; Anal. Calcd \% for $\left(\mathrm{C}_{12} \mathrm{H}_{13} \mathrm{~N}\right)$; C,84.59; H,9.68; N,5.80; Found: C,84.59; H,9.69; N,5.81.

1b.6-Chloro-2,3,4,9-tetrahydro-1H-carbazole

Yield 61.28\%; mp 121-123 ${ }^{\circ} \mathrm{C}$ (ethanol); Rf 0.67 (n - hexane: EtoAc,7:3); IR KBr cm${ }^{-1}: 3315.4$ (N-H stretching) ,2948.4 (C$\mathrm{H}$ Stretching aliphatic) ,1521.9 ( $\mathrm{C}=\mathrm{C}$ stretching),680.13 (C$\mathrm{Cl}$ stretching); ${ }^{1} \mathrm{HNMR}\left(\mathrm{CDCl}_{3}, 400 \mathrm{MHz}\right): 2.01\left(\mathrm{~d} .2 \mathrm{H}, \mathrm{CH}_{2}\right)$, 2.18(m,2H,ABq, $\left.\mathrm{CH}_{2}\right), 2.29\left(\mathrm{~m}, 4 \mathrm{H}, \mathrm{CH}_{2}\right), 3.12(\mathrm{~s}, 1 \mathrm{H}, \mathrm{NH})$, 6.83 (m,3H,Ar): ${ }^{13} \mathrm{CNMR}: 116.75,118.50,119.40$ (Ar), 169.00, 169.14, 170.22 (Tetrahydrocarbazole, Ar ): Em, (Es, Positive mode ) $\mathrm{m} / \mathrm{z} 205.02(\mathrm{~m}+\mathrm{H})$ : Anal. Calcd \% for $\left(\mathrm{C}_{12} \mathrm{H}_{12} \mathrm{ClN}\right)$; C,70.67; H,5.83; N,6.80, Found: C,70.68; H,5.84; N,6.81.

1c.6-Fluoro-2,3,4,9-tetrahydro-1H-carbazole

Yield $65.38 \%$; mp142 -143 ${ }^{\circ} \mathrm{C}$ (ethanol); Rf 0.68 (n - hexane : EtoAc,7:3); IR KBr cm${ }^{-1}: 317.12$ (N-H Stretching),2924.4 (C$\mathrm{H}$ stretching aliphatic), 1622.14( $\mathrm{C}=\mathrm{C}$ stretching), 1052.12(CF stretching); ${ }^{1} \mathrm{H} \mathrm{NMR}\left(\mathrm{CDCl}_{3}, 400 \mathrm{MHz}\right): 2.09$ (m, 2H, $\mathrm{ABq}$, $\left.\mathrm{CH}_{2}\right), 2.30\left(\mathrm{~m}, 2 \mathrm{H} \mathrm{ABq}, \mathrm{CH}_{2}\right), 2.79\left(\mathrm{~m}, 4 \mathrm{H}_{1} \mathrm{CH}_{2}\right), 3.90(\mathrm{~s}, 1 \mathrm{H}$, $\mathrm{NH}), 6.43(\mathrm{~m}, 3 \mathrm{H}, \mathrm{Ar}){ }^{13} \mathrm{C}$ NMR: 118.75, 119.50, 121.40 (Ar), 168.01, 169.14, 172.12 (TetrahydrocarbazoleAr); EM (ES, Positive mode): $\mathrm{m} / \mathrm{z} 189.126(\mathrm{~m}+\mathrm{H})^{+}$; Anal. Calcd \% for: $\left(\mathrm{C}_{12}\right.$ $\left.\mathrm{H}_{12} \mathrm{FN}\right)$; C,76.17; H,6.34; N,7.39; F,10.04: Found: C,76.18: H, 6.35; N,7.38.

1d.6-Methyl-2,3,4,9-tetrahydro-1H-carbazole

Yield 55\%; mp 212-284 ${ }^{\circ} \mathrm{C}$ (ethanol); Rf 0.76 (n-hexane: EtoAC, 7:3); IR KBr cm${ }^{-1}: 3329.12$ (N-H stretching),2884.4 (C-H stretching aliphatic),1622,67 ( $\mathrm{C}=\mathrm{C}$ stretching),738.78 $\left(\mathrm{C}-\mathrm{CH}_{3}\right) ;{ }^{1} \mathrm{H}$ NMR $\left(\mathrm{CDCl}_{3}, 400 \mathrm{MHz}\right): 1.90(\mathrm{~m}, 4 \mathrm{H}, \mathrm{ABq}$, $\left.\mathrm{CH}_{2}\right), 2.05\left(\mathrm{~m}, 2 \mathrm{H}, \mathrm{ABq}, \mathrm{CH}_{2}\right), 1.35\left(\mathrm{~m}, 3 \mathrm{H}, \mathrm{CH}_{3}\right), 3.92(\mathrm{~s}, \mathrm{H}$, $\mathrm{NH}), 2.30\left(\mathrm{~m}, 2 \mathrm{H}, \mathrm{ABq}, \mathrm{CH}_{2}\right), 6.53(\mathrm{~m}, 3 \mathrm{H}, \mathrm{Ar}) ;{ }^{13} \mathrm{C} \mathrm{NMR}$ $\left(\mathrm{CDCl}_{3}, 125 \mathrm{M} \mathrm{Hz}\right): 18.30\left(\mathrm{CH}_{3}\right), 22.30,30.15$ (aliphatic), 114.76, 119.89, 127.10 (Ar), 168.13, 170.12, 171.14 (TetrahydracarbazoleAr); EM(ES positive mode): $\mathrm{m} / \mathrm{z} 185.124$ $(\mathrm{m}+\mathrm{H})^{+}$; Anal. calcd \% for: $\left(\mathrm{C}_{12} \mathrm{H}_{15} \mathrm{~N}\right)$; C,84.28; H,8.63; N,7.55: Found: C, 84.29; H, 8.64; N, 7.56. 2a: 9-[(4-methylphenyl)sulfonyl]-2,3,4,9-tetrahydro- $1 H$ carbazole

Yield 61\%;mp 322- $323{ }^{\circ} \mathrm{C}$ (ethanol); Rf 0.86 (n-hexane EtoAC, 7:3) ; IR $\mathrm{KBrcm}^{-1}: 3018.45(\mathrm{C}-\mathrm{H}$ aromatic Stretching) ,2856.78 (C-H aliphatic stretching),1601.78 (C=C aromatic stretching), $1346.67(\mathrm{O}=\mathrm{S}=\mathrm{O}$ Stretching $),{ }^{1} \mathrm{H}$ NMR $\left(\mathrm{CDCl}_{3}, 400 \mathrm{MHz}\right) ; 2.28\left(\mathrm{~m}, 4 \mathrm{H}, \mathrm{CH}_{2}\right), 2.08(\mathrm{~m}, 2 \mathrm{H}, \mathrm{ABq}$, $\left.\mathrm{CH}_{2}\right), 1.35\left(\mathrm{t}, 3 \mathrm{H}, \mathrm{CH}_{3}\right), 2.20\left(\mathrm{~m}, 2 \mathrm{H}, \mathrm{ABq}, \mathrm{CH}_{2}\right), 6.83(\mathrm{~m}, 4 \mathrm{H}$, $\mathrm{Ar}), 7.95(\mathrm{~m}, 4 \mathrm{H}, \mathrm{Ar}) ;{ }^{13} \mathrm{CNMR}\left(\mathrm{CDCl}_{3}, 125 \mathrm{MHz}\right) ; 17.30$, 18.90, $\left(\mathrm{CH}_{3}\right), 21.96,22.30,30.15$ (aliphatic), 114.76, 117.89, 127.10 (Ar), 167.13, 170.12, 171.14 (Tetrahydracarbazole Ar). EM (ES positive mode); $\mathrm{m} / \mathrm{z} 328.76(\mathrm{~m}+\mathrm{H})^{+}$.Anal. calcd for $\left(\mathrm{C}_{19} \mathrm{H}_{19} \mathrm{NO}_{2} \mathrm{~S}\right) ; \mathrm{C}, 70.70 ; \mathrm{H}, 5.83 ; \mathrm{N}, 4.30$ : Found: $\mathrm{C}, 70.29$; $\mathrm{H}, 5.64 ; \mathrm{N}, 4.56$.

2b: 6-chloro-9-[(4-methylphenyl)sulfonyl]-2,3,4,9-tetrahydro-1H-carbazole

Yield 61\%; mp : 338-340 ${ }^{\circ} \mathrm{C}$ (ethanol); Rf 0.91 (n-hexane EtoAC,7:3) IR KBr cm${ }^{-1}: 2950.68$ (C-Haromatic stretching), 2876.98 (C-H aliphatic stretching), 1627.34 (C=C aromatic stretching $), \quad 1334.74 \quad(\mathrm{O}=\mathrm{S}=\mathrm{O}$ Stretching $), \quad 735.78(\mathrm{C}-\mathrm{Cl}$ stretching) : ${ }^{1} \mathrm{H} \mathrm{NMR}\left(\mathrm{CDCl}_{3}, 400 \mathrm{M} \mathrm{Hz}\right) ; 2.21\left(\mathrm{~m}, 4 \mathrm{H}, \mathrm{CH}_{2}\right)$, $2.08\left(\mathrm{~m}, 2 \mathrm{H}, \mathrm{ABq}, \mathrm{CH}_{2}\right)$ 1. $25\left(\mathrm{t}, 3 \mathrm{H}, \mathrm{CH}_{3}\right), 2.10$ $\left(\mathrm{m}, 2 \mathrm{H}, \mathrm{ABq}, \mathrm{CH}_{2}\right), 6.23$ (m 3H , Ar ), 7.85 (m, 4H,Ar) ${ }^{13} \mathrm{C}$ NMR ( $\left.\mathrm{CDCl}_{3}, 125 \mathrm{M} \mathrm{Hz}\right) 16.90,17.56,18.30 \quad\left(\mathrm{CH}_{3}\right)$, 22.30,30.15( aliphatic), 114.96, 116.89, 128.10 (Ar) 166.13, 169.12, 171.15 ( Tetrahydracarbazole Ar). EM (ES positive mode $) ; \mathrm{m} / \mathrm{z} 358.76(\mathrm{~m}+\mathrm{H})^{+}$.Anal. calcd for $\left(\mathrm{C}_{19} \mathrm{H}_{18} \mathrm{ClNO}_{2} \mathrm{~S}\right)$; C,63.70; H,5.00;N,3.30.Found: C, 63.79; H,5.04; N,4.91.

\section{2c: 6-fluoro-9-[(4-methylphenyl) sulfonyl]-2,3,4,9-tetrahy- dro- $1 \mathrm{H}$-carbazole}

Yield 63\%;mp 342- $343^{\circ} \mathrm{C}$ ( ethanol); Rf 0.98 (n-hexane EtoAC,7:3); IR $\mathrm{KBrcm}^{-1}: 3097.56 \mathrm{C}-\mathrm{H}$ aromatic stretching),2987.56 (C-H aliphatic stretching),1464.12(O =S = O Stretching ), 1598.35( C=C aromaticstretching) ,1143.79(C-F stretching): ${ }^{1} \mathrm{H}$ NMR ( $\left.\mathrm{CDCl}_{3}, 400 \mathrm{MHz}\right) ; 2.16\left(\mathrm{~m}, 4 \mathrm{H}_{1} \mathrm{CH}_{2}\right)$, $2.18\left(\mathrm{~m}, 2 \mathrm{H}, \mathrm{ABq}, \mathrm{CH}_{2}\right) 1.35\left(\mathrm{t}, 3 \mathrm{H}, \mathrm{CH}_{3}\right), 2.60(\mathrm{~m}, 2 \mathrm{H}, \mathrm{ABq}$, $\left.\mathrm{CH}_{2}\right), 6.78(\mathrm{~m} \mathrm{3H}, \mathrm{Ar}), 7.25(\mathrm{~m}, 4 \mathrm{H}, \mathrm{Ar}) ;{ }^{13} \mathrm{C} \mathrm{NMR}\left(\mathrm{CDCl}_{3}\right.$, $125 \mathrm{M} \mathrm{Hz})$ 16.30.17.89 ( $\left.\mathrm{CH}_{3}\right), 22.30,23.67,30.15$ (aliphatic), 114.76, 119.89, 128.10 (Ar) 165.13, 169.12, 171.84 ( Tetrahydracarbazole Ar). EM (ES positive mode); m/z 344.76 $(\mathrm{m}+\mathrm{H})^{+}$.Anal. calcd for $\left(\mathrm{C}_{19} \mathrm{H}_{18} \mathrm{FNO}_{2} \mathrm{~S}\right) ; \mathrm{C}, 66.45 ; \mathrm{H}, 5.23 ; \mathrm{N}$ 4.07; Found: C, 66.49; H,5.24; $\mathrm{N}, 4.06$.

\section{2d: 6-methyl-9-[(4-methylphenyl)sulfonyl]-2,3,4,9-tetra- hydro- $1 H$-carbazole}

Yield 61\%; mp 346- $348^{\circ} \mathrm{C}$ (ethanol); Rf 0.91(n-hexane EtoAC 7:3); IR KBrcm-1:3111.18(C-H aromatic Stretching), 2956.13 (C-H aliphatic stretching), 1537.98 (C=C aromatic stretching), 1426.87( $\mathrm{O}=\mathrm{S}=$ OStretching), 924.24(C-

$\mathrm{CH}_{3}$ Stretching); ${ }_{1} \mathrm{HNMR} \quad\left(\mathrm{CDCl}_{3}, \quad 400 \mathrm{MHz}\right)$; $2.35\left(\mathrm{~m}, 4 \mathrm{H}, \mathrm{CH}_{2}\right), 2.38\left(\mathrm{~m}, 2 \mathrm{H}, \mathrm{ABq}, \mathrm{CH}_{2}\right) 1.72\left(\mathrm{t}, 3 \mathrm{H}, \mathrm{CH}_{3}\right), 1.25$ $\left(\mathrm{t}, 3 \mathrm{H}, \mathrm{CH}_{3}\right), 2.20\left(\mathrm{~m}, 2 \mathrm{H}, \mathrm{ABq}, \mathrm{CH}_{2}\right), 6.73(\mathrm{~m} 3 \mathrm{H}, \mathrm{Ar}), 7.35$ $(\mathrm{m}, 4 \mathrm{H}, \mathrm{Ar}){ }^{13} \mathrm{C} \mathrm{NMR}\left(\mathrm{CDCl}_{3}, 125 \mathrm{M} \mathrm{Hz}\right) 17.34,19.30\left(\mathrm{CH}_{3}\right)$, 22.30,24.37, 30.15(aliphatic), 114.76, 124.89, 127.10 (Ar), 168.13, 169.12, 171.14 ( Tetrahydracarbazole Ar). EM (ES positive mode); m/z $339.76(\mathrm{~m}+\mathrm{H})^{+}$.Anal. calcd for $\left(\mathrm{C}_{20}\right.$ $\left.\mathrm{H}_{21} \mathrm{FNO}_{2} \mathrm{~S}\right) ; \mathrm{C}, 70.45 ; \mathrm{H}, 6.23 ; \mathrm{N}, 4.12$ : Found: $\mathrm{C}, 70.49 ; \mathrm{H}, 6.24$; $\mathrm{N}, 4.13$.

\section{3a.(4-chlorophenyl)(1,2,3,4-tetrahydro-9H-carbazol-9- yl)methanone}

Yield : $64.12 \%$ : mp : $256-258^{\circ} \mathrm{C}$ (Ethanol ): Rf : 0.54 (n- hexanol : EtoAC 7:3): IR $\mathrm{KBrcm}^{-1}$

:3052.67 (C-H aromatic Stretching ) ,2967.35(C-H aliphatic strectching), $1702.78 \quad(\mathrm{C}=\mathrm{O}$ stretching) ,1623.89 (C=C stretching Ar), 739.38 (C-Cl stretching): ${ }^{1} \mathrm{H} \mathrm{NMR}\left(\mathrm{CDCl}_{3}\right.$, 
Table 1: Docking scores of the synthesized compounds.

\begin{tabular}{cccc}
\hline S1. No. & Ligands & Docking scores & Hydrogen bond interactions \\
\hline 1 & ciprofloxacine & -3.5399 & Met28A-Pro29A-Leu30A-Thr36A-VAL31A \\
2 & $1 \mathrm{a}$ & -4.4808 & Pro29A-LEU30A-Val31A-Thr36A-ILE37A-SER35A \\
3 & $1 \mathrm{~b}$ & -4.6162 & Leu30A-val31A-Ser35A-Thr36A-Ile37A \\
4 & $1 \mathrm{c}$ & -4.5488 & Met28A-Pro29A-Val31A-Cys38A \\
5 & $1 \mathrm{~d}$ & -4.2528 & Met28A-Pro29A-Leu30A \\
6 & $2 \mathrm{a}$ & -4.2017 & Leu30A-val31A-Ser35A-Thr36A-Ile37A \\
7 & $2 \mathrm{~b}$ & -4.3146 & Pro29A-LEU30A-Val31A-Thr36A-ILE37A \\
8 & $2 \mathrm{c}$ & -0.7244 & Met28A-Ser35A \\
9 & $2 \mathrm{~d}$ & -4.8527 & Met28A-Pro29A-Val31A-Cys38A \\
10 & $3 \mathrm{a}$ & 0.9109 & Met28A-Ser35A \\
11 & $3 \mathrm{~b}$ & -4.3018 & Pro29A-LEU30A-Val31A-Thr36A-ILE37A \\
12 & $3 \mathrm{c}$ & -2.1018 & Met28A-Ser35A-Ile37A \\
13 & $3 \mathrm{~d}$ & -2.2002 & Pro29A-LEU30A-Val31A-Thr36A-ILE37A \\
14 & $3 \mathrm{e}$ & -1.7063 & LEU30A-Val31A-Thr36A-ILE37A \\
15 & $3 \mathrm{f}$ & -0.9294 & LEU30A-Val31A-Thr36A-ILE37A \\
16 & $3 \mathrm{~g}$ & -1.8973 & Met28A-Ser35A \\
\hline
\end{tabular}

Table 2: Anti-microbial activity of synthesized compounds.

\begin{tabular}{|c|c|c|c|c|c|c|c|}
\hline \multirow[b]{2}{*}{ S1. No. } & \multirow[b]{2}{*}{ Ligands } & \multicolumn{6}{|c|}{ Zone of inhibitions in mm. } \\
\hline & & $\begin{array}{c}\text { E. coli } \\
\text { ATCC } 25922\end{array}$ & $\begin{array}{c}\text { S. aureus } \\
\text { ATCC } 29213\end{array}$ & $\begin{array}{l}\text { P. aeruginosa } \\
\text { ATCC27953 }\end{array}$ & $\begin{array}{c}\text { B. subtilis } \\
\text { ATCC } 6633\end{array}$ & $\begin{array}{l}\text { C. albucans } \\
\text { NRRC } 477\end{array}$ & A. niger \\
\hline 1 & $1 \mathrm{a}$ & 18 & 16 & 13 & 11 & 10 & 13 \\
\hline 2 & $1 b$ & 17 & 18 & 14 & 13 & 11 & 16 \\
\hline 3 & 1c & 16 & 17 & 16 & 14 & 10 & 15 \\
\hline 4 & $1 d$ & 15 & 16 & 12 & 12 & 13 & 10 \\
\hline 5 & $2 \mathrm{a}$ & 16 & 17 & 11 & 11 & 9 & 12 \\
\hline 6 & $2 b$ & 14 & 16 & 14 & 8 & 14 & 9 \\
\hline 7 & $2 c$ & 15 & 13 & 10 & 13 & 12 & 10 \\
\hline 8 & $2 d$ & 18 & 14 & 12 & 10 & 14 & 11 \\
\hline 9 & $3 a$ & 14 & 16 & 11 & 8 & 10 & 13 \\
\hline 10 & $3 b$ & 13 & 14 & 10 & 9 & 13 & 12 \\
\hline 11 & $3 c$ & 15 & 8 & 12 & 11 & 11 & 9 \\
\hline 12 & $3 d$ & 14 & 16 & 14 & 9 & 12 & 10 \\
\hline 13 & $3 e$ & 18 & 14 & 11 & 10 & 11 & 11 \\
\hline 14 & $3 f$ & 11 & 15 & 13 & 8 & 11 & 10 \\
\hline 15 & $3 g$ & 12 & 14 & 11 & 16 & 10 & \\
\hline 16 & solvent & - & - & - & - & - & - \\
\hline 17 & Ciprofloxacin & 23 & 22 & 21 & 23 & - & - \\
\hline 18 & Fluconazole & - & - & - & - & 22 & 21 \\
\hline
\end{tabular}

$400 \mathrm{MHz}): 2.09\left(\mathrm{~m}, 2 \mathrm{H}, \mathrm{CH}_{2}\right), 2.21\left(\mathrm{~m}, 2 \mathrm{H}, \mathrm{CH}_{2}\right), 2.98$ (m.4H, CH $), 6.73$ ( m. 3H,Ar) , $7.36(\mathrm{~m}, 5 \mathrm{H}, \mathrm{Ar}):{ }^{13} \mathrm{C}$ NMR $\left(\mathrm{CDCl}_{3}, 125 \mathrm{MHz}\right): 119.76,120.80,121.70,129.13,133.82(\mathrm{Ar})$ 167.13, 169.14, 171.13 ( tetrahydrocarbazole (Ar) , 192.90 $(\mathrm{C}=\mathrm{O}$ ): Em (Es, Positive mode ) m/z 309.31: Anal, Calc for( $\left.\mathrm{C}_{19} \mathrm{H}_{16} \mathrm{NO}\right)$ : C,73.58; H,5.16; N,7.99; F,4.41: Found: C,72.57; H,5.43; N,7.98.

\section{3b.(4-chlorophenyl)(6-chloro-1,2,3,4-tetrahydro-9H-car- bazol-9-yl)methanone}

Yield : $55.23 \%$ : mp : $260-262^{\circ} \mathrm{C}$ (Ethanol ): Rf : 0.58 (n- hexanol : EtoAC 7:3): IR KBrcm ${ }^{-1}$

:3109.78 (C-H aromatic Stretching), 2993.01(C-H aliphatic stretching),1748.12 ( $\mathrm{C}=\mathrm{O}$ stretching) ,1615.34 (C=C stretching $\mathrm{Ar}$, ,780.15,736.12 (C-Cl stretching): ${ }^{1} \mathrm{H}$ NMR ( $\mathrm{CDCl}_{3}$, $400 \mathrm{MHz}): 2.10\left(\mathrm{~m}, 2 \mathrm{H}, \mathrm{CH}_{2}\right), 2.32(\mathrm{~m}, 2 \mathrm{H}$, $\left.\mathrm{CH}_{2}\right), 3.78\left(\mathrm{~m}, 4 \mathrm{H}_{1} \mathrm{CH}_{2}\right), 6.24$ ( m. 3H, Ar) , $7.89(\mathrm{~m}, 4 \mathrm{H}, \mathrm{Ar})$ : ${ }^{13} \mathrm{C}$ NMR ( $\left.\mathrm{CDCl}_{3}, 125 \mathrm{MHz}\right): 119.71,120.89,121.74,129.19$, 133.84 (Ar), 167.16, 169.19, 171.15 ( tetrahydrocarbazole (Ar) , 192.98 (C=O ): Em (Es, Positive mode ) m/z 345.34: Anal, Calc for $\left(\mathrm{C}_{19} \mathrm{H}_{15} \mathrm{Cl}_{2} \mathrm{NO}\right)$ : C, 66.28; $\mathrm{H}, 4.35 ; \mathrm{N}, 4.06$ : Found: C,66.89; H,4.78; N,4.89.
3c.(4-chlorophenyl)(6-fluoro-1,2,3,4-tetrahydro-9H-carbazol-9-yl)methanone

Yield : $59.67 \%$ : mp : $264-266^{\circ} \mathrm{C}$ (Ethanol ): Rf : 0.60 (n- hexanol : EtoAC 7:3): IR KBrcm ${ }^{-1}$

:3111.18 (C-H aromatic Stretching) ,2943.37(C-H aliphatic stretching),1729.65 ( $\mathrm{C}=\mathrm{O}$ stretching) ,1615.36 ( $\mathrm{C}=\mathrm{C}$ stretching $\mathrm{Ar}), 1130.29$ (C-F stretching),745.23 (C-Cl stretching): $\mathrm{H} \mathrm{NMR}\left(\mathrm{CDCl}_{3}, 400 \mathrm{MHz}\right): 2.08\left(\mathrm{~m}, 2 \mathrm{H}, \mathrm{CH}_{2}\right), 2.32$ ( m, $\left.2 \mathrm{H}, \mathrm{CH}_{2}\right), 2.86\left(\mathrm{~m}, 4 \mathrm{H}, \mathrm{CH}_{2}\right), 6.24(\mathrm{~m} .3 \mathrm{H}, \mathrm{Ar}), 7.89(\mathrm{~m}, 4 \mathrm{H}$, $\mathrm{Ar}):{ }^{13} \mathrm{C}$ NMR ( $\left.\mathrm{CDCl}_{3}, 125 \mathrm{MHz}\right): 119.78,120.84,121.754$, 129.23, 133.67 (Ar), 167.45, 169.87, 171.23 (tetrahydrocarbazole (Ar) , 192.13 (C=O ): Em (Es, Positive mode ) m/z 328.45: Anal, Calc for $\left(\mathrm{C}_{19} \mathrm{H}_{15} \mathrm{ClFNO}\right): \mathrm{C}, 69.61 ; \mathrm{H}, 5.49$; N,4.32: Found: C,69.78; H,5.89; N,4.69.

3d. (4-chlorophenyl)(6-methyl-1,2,3,4-tetrahydro-9H-carbazol-9-yl)methanone

Yield: $54.78 \%$ : mp: $272-274^{\circ} \mathrm{C}$ (Ethanol): $\mathrm{R}_{\mathrm{f}}: 0.59$ (n- hexanol : EtoAC 7:3): IR KBrcm ${ }^{-1}$

:3052.41 (C-H aromatic Stretching) ,2927.56(C-H aliphatic stretching),1742.56 ( $\mathrm{C}=\mathrm{O}$ stretching) ,1617.98 (C=C stretching $\mathrm{Ar}$ ) ,1423.56 (C-CH3 stretching),765.23 (C-Cl stretching): 
${ }^{1} \mathrm{H} \mathrm{NMR}\left(\mathrm{CDCl}_{3}, 400 \mathrm{MHz}\right): 2.45\left(\mathrm{~m}, 2 \mathrm{H}, \mathrm{CH}_{2}\right), 2.57$ ( m, $\left.2 \mathrm{H}, \mathrm{CH}_{2}\right), 2.89\left(\mathrm{~m}, 4 \mathrm{H}, \mathrm{CH}_{2}\right), 6.16(\mathrm{~m} .3 \mathrm{H}, \mathrm{Ar}), 7.45(\mathrm{~m}, 4 \mathrm{H}$, $\mathrm{Ar}):{ }^{13} \mathrm{C} \mathrm{NMR}\left(\mathrm{CDCl}_{3}, 125 \mathrm{MHz}\right): 119.68,120.48,121.28$, 129.89, 133.689 (Ar), 167.29, 169.20, 171.219 ( tetrahydrocarbazole (Ar) , 192.78 (C=O ): Em (Es, Positive mode ) m/z 324.89: Anal, Calc for $\left(\mathrm{C}_{20} \mathrm{H}_{18} \mathrm{NClO}\right)$ : C, 74.17; $\mathrm{H}, 5.55 ; \mathrm{N}, 4.31$ : Found: C,75.89; H,5.78; N,4.79.

3e. (6-chloro-1,2,3,4-tetrahydro-9H-carbazol-9-yl)(4-nitrophenyl)methanone

Yield : $60.15 \%$ : mp : $278-280^{\circ} \mathrm{C}$ (Ethanol ): Rf : 0.76 (n- hexanol : EtoAC 7:3): IR KBrcm ${ }^{-1}$

:3167.45 (C-H aromatic Stretching) ,2978.56(C-H aliphatic stretching),1726.89 ( $\mathrm{C}=\mathrm{O}$ stretching) ,1660.57 ( $\mathrm{C}=\mathrm{C}$ stretching Ar) 1356.23 (C-NO2 stretching),756.46 ( C-Cl stretching): ${ }^{1} \mathrm{H}$ NMR $\left(\mathrm{CDCl}_{3}, 400 \mathrm{MHz}\right): 2.56(\mathrm{~m}, 2 \mathrm{H}$, $\left.\mathrm{CH}_{2}\right), 2.78\left(\mathrm{~m}, 2 \mathrm{H}, \mathrm{CH}_{2}\right), 2.89\left(\mathrm{~m}, 4 \mathrm{H}, \mathrm{CH}_{2}\right), 6.54(\mathrm{~m}$. $3 \mathrm{H}, \mathrm{Ar}), 7.16(\mathrm{~m}, 4 \mathrm{H}, \mathrm{Ar}):{ }^{13} \mathrm{C} \mathrm{NMR}\left(\mathrm{CDCl}_{3}, 125\right.$ MHz) :119.18, 120.78, 121.24, 129.78, 133.94 (Ar), 167.25, 169.67, 171.79 ( tetrahydrocarbazole (Ar) , 192.74 (C=O ): Em (Es, Positive mode) m/z 355.68s: Anal, Calc for $\left(\mathrm{C}_{19} \mathrm{H}_{15} \mathrm{ClN}_{2} \mathrm{O}_{3}\right)$ : C, 64.31; H,4.22; N,7.89: Found: C,65.34; $\mathrm{H}, 4.89 ; \mathrm{N}, 7.43$.

3f. (6-fluoro-1,2,3,4-tetrahydro-9H-carbazol-9-yl)(4-nitrophenyl)methanone

Yield: $54.98 \%$ : mp : $280-282^{\circ} \mathrm{C}$ (Ethanol ): Rf : 0.78 (n- hexanol : EtoAC 7:3): IR KBrcm ${ }^{-1}$

:3121.89 (C-H aromatic Stretching) ,2987.47(C-H aliphatic stretching), 1710.14 ( $\mathrm{C}=\mathrm{O}$ stretching) ,1635.76 ( $\mathrm{C}=\mathrm{C}$ stretching $\mathrm{Ar}), 1335.67 \quad\left(\mathrm{C}-\mathrm{NO}_{2}\right.$ stretching $), 746.35$ ( $\mathrm{C}-\mathrm{Cl}$ stretching) : ${ }^{1} \mathrm{H}$ NMR ( $\left.\mathrm{CDCl}_{3}, 400 \mathrm{MHz}\right): 2.34(\mathrm{~m}, 2 \mathrm{H}$, $\left.\mathrm{CH}_{2}\right), 2.57\left(\mathrm{~m}, 2 \mathrm{H}, \mathrm{CH}_{2}\right), 2.92\left(\mathrm{~m}, 4 \mathrm{H}, \mathrm{CH}_{2}\right), 6.16(\mathrm{~m}$. $3 \mathrm{H}, \mathrm{Ar}), 7.27(\mathrm{~m}, 4 \mathrm{H}, \mathrm{Ar}):{ }^{13} \mathrm{C} \mathrm{NMR}\left(\mathrm{CDCl}_{3}, 125\right.$ MHz) :119.19, 120.28, 121.24, 129.39, 133.29 (Ar), 167.36, 169.28, 171.98 ( tetrahydrocarbazole (Ar) , 192.89 (C=O ): Em (Es, Positive mode ) $\mathrm{m} / \mathrm{z}$ 339.45: Anal, Calc for $\left(\mathrm{C}_{19} \mathrm{H}_{15} \mathrm{FN}_{2} \mathrm{O}_{3}\right)$ : $\mathrm{C}, 67.44 ; \mathrm{H}, 4.43 ; \mathrm{N}, 8.27$ : Found: $\mathrm{C}, 67.34$; $\mathrm{H}, 4.80 ; \mathrm{N}, 8.49$. 3g. (6-nitro-1,2,3,4-tetrahydro-9H-carbazol-9-yl)(4-nitrophenyl)methanone

Yield : $57.45 \%$ : $\mathrm{mp}: 282-284^{\circ} \mathrm{C}$ (Ethanol ): $\mathrm{R}_{\mathrm{f}}: 0.80$ (n- hexanol : EtoAC 7:3): IR $\mathrm{KBrcm}^{-1}$

:3135.35 (C-H aromatic Stretching) ,2927.46(C-H aliphatic stretching),1708.34 ( $\mathrm{C}=\mathrm{O}$ stretching) ,1618.34 (C=C stretching $\mathrm{Ar}), 1435.24,1337.89$. (C-NO2 $\mathrm{N}_{2}$ stretching),746.35 : ${ }^{1} \mathrm{H}$ $\mathrm{NMR}\left(\mathrm{CDCl}_{3}, 400 \mathrm{MHz}\right): 2.16\left(\mathrm{~m}, 2 \mathrm{H}, \mathrm{CH}_{2}\right), 2.35(\mathrm{~m}, 2 \mathrm{H}$, $\left.\mathrm{CH}_{2}\right), 2.39\left(\mathrm{~m}, 4 \mathrm{H}, \mathrm{CH}_{2}\right), 6.25(\mathrm{~m} .3 \mathrm{H}, \mathrm{Ar}), 7.78(\mathrm{~m}, 4 \mathrm{H}, \mathrm{Ar})$ : ${ }^{13} \mathrm{C}$ NMR ( $\left.\mathrm{CDCl}_{3}, 125 \mathrm{MHz}\right): 119.89,120.57,121.45,129.47$, 133.69 (Ar), 167.37, 169.69, 171.35 ( tetrahydrocarbazole (Ar) , 192.25 ( $\mathrm{C}=\mathrm{O}$ ): Em (Es, Positive mode ) m/z 366.89: Anal, Calc for $\left(\mathrm{C}_{19} \mathrm{H}_{15} \mathrm{~N}_{3} \mathrm{O}_{5}\right): \mathrm{C}, 62.46 ; \mathrm{H}, 4.10 ; \mathrm{N}, 11.49$ : Found: C,63.67; $\mathrm{H}, 4.56 ; \mathrm{N}, 11.78$.

\section{Docking studies}

Substituted 1,2,3,4- tetrahydrocarbazoles were subjected to molecular docking studies to the target enzyme GlcN-6-P, Code 1 XFF by using V life MDS software. The compounds shown good interaction with the enzyme. The enzyme 1XFF is having 9 active pockets and the ligands showed good interaction with $2^{\text {nd }}$ active pocket. The structure of GlcN-6-P was showed in figure 3. The interaction of ligands and selective inhibition in terms of docking scores (binding energies) is depicted in the table 1 . The ligands showed hydrogen bonding, Van der Waals forces, Charge, hydrophobic interactions with the enzyme. The amino acids that interact with GlcN-6-P in most of the ligands are MET28A, PRO29A, LEU 30A, VAL31A, SER35A, THR36A ILE37A, CYS38. The hydrogen bond between receptor and ligand is observed to be $3.425-4.135 \AA$.

\section{Anti-microbial activity}

All the newly synthesized compounds were screened for their anti-bacterial activity against S. aureus, E. coli, B. subtilis, P. aeruginosa and Antifungal activity for C. albicans, A. niger. The compounds showed marked zone of inhibition. The different spectrum of activity by the test compounds against the tested microorganisms may be due to different
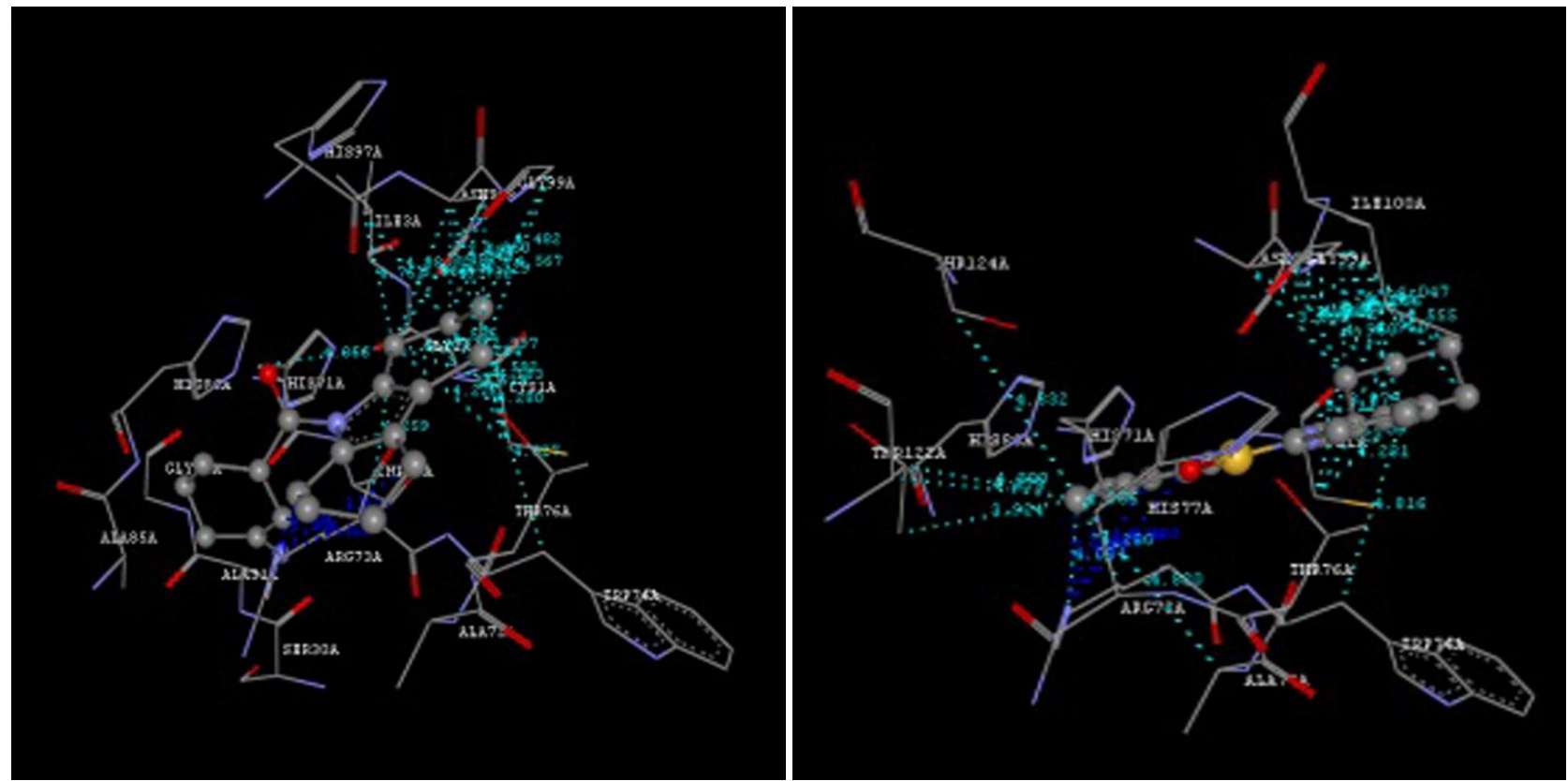

Figure 3: Docked complex with the Gln-6p Enzyme. 
substituents present in the substituted tetrahydrocarbazoles. The results of the anti-microbial activity were showed in table 2 .

A series of substituted tetrahydrocarbazole derivatives were synthesized in well manner with respect to percentage yield and all the compounds are subjected for anti-microbial studies. The synthesized tetrahydrocarbazole compounds was projected to analytical techniques of FT-IR, ${ }^{1} \mathrm{H}-\mathrm{NMR},{ }^{13} \mathrm{C}$ NMR, Mass Spectrometry for confirming their chemical structure. The studies of the docking were carried out using V-Life MDS software for all the derivatives. The derivatives compounds were docked with target enzyme GlcN-6-P synthase. The results of docking scores were shown in the Table 1 among all the docked compounds $1 \mathrm{a}, 1 \mathrm{~b}, 1 \mathrm{c}, 1 \mathrm{~d}, 2 \mathrm{a}, 2 \mathrm{~b}, 2 \mathrm{~d}, 3 \mathrm{~b}$ showed good binding affinity and interaction with enzyme with reference to ciprofloxacin and flucanazole. The docking results suggests that the parameters for docking simulation are optimum in producing experimental orientation of these compounds. The pharmacological study was undertaken to evaluate the effect of substituent on the anti-microbial activity. All the synthesized tetrahydrocarbazoles showed prominent anti-microbial activity.

\section{CONCLUSION}

Fifteen new tetrahydrocarbazole derivatives were synthesized and were characterized by ${ }^{1} \mathrm{H} \mathrm{NMR},{ }^{13} \mathrm{C}$ NMR, Mass spectrometry, FT-IR studies and elemental analysis. The newly synthesized tetrahydrocarbazole derivatives were studied for anti-microbial activity using agar cup method. In silico studies exposed that all the synthesized compounds have relatively better binding affinity as compared to the standard drug. So, it may be considered as a good inhibitor of GlcN-6-P. The compounds are subjected to antimicrobial activity, out of fifteen compounds, eight compounds are showed good zone of inhibition. Hence, this study has widened the scope of developing these tetrahydrocarbazoles derivatives as promising anti-microbial agents.

\section{ACKNOWLEDGEMENT}

The authors are thankful to the Microbiology Department, Nagpur University, Institute of Microbial Technology, Chandigarh, India, Loyola intex laboratories, Vijayawada, Andhra Pradesh, India and NRK \& KSR Gupta College of pharmacy, Tenali, Andhra Pradesh, India for providing the support to carry some part of the research work. The authors are thanked to Mr. K. Pavan Kumar, Asst. Professor, Dept. of Pharmaceutical Analysis and Quality Assurance, Raghavendra Institute of Pharmaceutical Education and Research (RIPER), Anantapur, Andhra Pradesh, India for proof reading of the work.

\section{REFERENCES}

Biswanath C., Suchandra, C., and Chandan, S. (2014). Antibacterial Activity of Murrayaquinone A and 6-Methoxy-3,7-dimethyl-2,3-dihydro- $1 \mathrm{H}$ carbazole-1,4(9H)-dione. International Journal of Microbiology, Volume 2014, Pages 1-8

Chakraborty, B., Chakraborty, S., and Saha, C. (2014). Antibacterial Activity of Murrayaquinone A and 6-Methoxy-3,7-dimethyl-2,3-dihydro- $1 \mathrm{H}$ carbazole-1,4(9H)-dione. International Journal of Microbiology, Volume 8, Pages 1-8. [DOI]

Colin, D., and William RP. (2006). Serum lipid-lowering properties of 6chloro-9-[2-(6-methyl-3-pyridyl)ethyl]-1,2,3,4-tetrahydrocarbazole-2carboxylic acid. Journal of Pharmaceutical Sciences, Volume 66, Issue 03, Pages 348-352.

Conchon, E., Anizon, F., Aboab, B., Golsteyn, RM., Leonce, S., Pfeiffer, B. and Prudhomme, M. (2008). Synthesis, in vitro antiproliferative activities, and Chk1 inhibitory properties of pyrrolo[3,4-a]carbazole-1,3diones, pyrrolo[3,4-c]carbazole-1,3-diones, and 2-aminopyridazino[3,4a]pyrrolo[3,4-c]carbazole-1,3,4,7-tetraone. European Journal of Medicinal Chemistry, Volume 43, Issue 2, Pages 282-292. [DOI]

Crosby, U., Rogers, BB., and Corson, J. (1947). One-Step Synthesis of 1,2,3,4-Tetrahydrocarbazole and 1,2-Benzo-3,4-dihydrocarbazole. Journal of American Chemical Society, Volume 69, Issue 11, Pages 29102911.

Hughes, JP., Rees, S., Kalindjian, SB. and Philpott, KL. (2011). Principles of early drug discovery. British Journal of Pharmacology, volume 162, Issue 6, Pages 1239-1249. [DOI]

Kapetanovic, IM. (2008). Computer-Aided Drug Discovery and Development (CADDD): in silico-chemico-biological approach. Chemico Biological Interactions, Volume 171, Issue 2, Pages 165-176. [DOI]

Kaushik, K., Kumar, N., and Pathak, D. (2012). Synthesis of some newer carbazole derivatives and evaluation for their pharmacological activity. Der Pharmacia Sinica, Volume 3, Issue 4, Pages 470-478.

Kumar, R., Ramachandran, U., Srinivasan, K., Ramarao, P., Raichur, S., and Chakrabarti, R. (2005). Design, synthesis and evaluation of carbazole derivatives as PPAR $\alpha / \gamma$ dual agonists and antioxidants. Bioorganic and Medicinal Chemistry, Volume 13, Issue 13, Pages 4279-4290. [DOI]

Meng, XY., Zhang, HX., Mezei, M., and Meng, C. (2011). Molecular Docking: A powerful approach for structure-based drug discovery. Current Computer Aided Drug Design, Volume 7, Issue 2, Pages 146-157. [DOI]

Rajesh, BP., and Sanjay, DS. (2015). Synthesis, characterization, molecular docking and evaluation of antimicrobial and antiproliferative properties of 3-substituted chromen-2-one derivatives. Der Pharma Chemica, Volume 7, Issue 3, Pages 26-37.

Subramanyam, L., Vaikuntara,o L., and Eswararao, B. (2014). Design, synthesis method development validation of $\mathrm{N}$-substituted

tetrahydrocarbazoles for analgesic, anti-inflammatory activity. Asian Journal of Pharmaceutical and Clinical Research, Volume 2, Issue 2, Pages 152-155.

Vijesh, AM., Arun, MI., Sandeep, T., Arulmoli, T., and Hoong, KF. (2013). Molecular docking studies of some new imidazole derivatives for antimicrobial properties. Arabian Journal of Chemistry, Volume 6, Issue 2, Pages 197-204. [DOI]

Ya-Ching, S., Ching-Yen, C., Pei-Wen, H., Chang-Yih, D., Yat-Min, L., and Chin-Lein, Ko. (2005). The preparation and evaluation of 1-Subsituted 1,2,3,4-tetrahydro- and 3,4-Dihydro- $\beta$-carboline derivatives as potential Antitumor agents. Chemical and Pharmaceutical Bulletin, Volume 53, Issue 1, Pages 32-36.

Zhang, FF., Gan, LL., and Zhou, CH. (2010). Synthesis, antibacterial and antifungal activities of some carbazole derivatives. Bioorganic and Medicinal Chemistry Letters, Volume 20, Issue 24, Pages 1881-1884. [DOI]

Zhu, G., Conner, SE., Zhou, X., Chan, HK., Shih, C., Engler, TA., Al-awar, RS., Brooks, HB., Watkins, SA., Spencer, CD., Schultz, RM., Dempsey, JA., Considine, EL., Patel, BR., Ogg, CA., Vasudevan, V., and Lytle, ML. (2004). Synthesis of 1,7-annulated indoles and their applications in the studies of cyclin dependent kinase inhibitors. Bioorganic \& Medicinal Chemistry Letters, Volume 14, Issue 12, Pages 3057-3061. [DOI] 